\title{
Research of Supporting Ability and Prediction of Residual Life of Casing Pipes at General Corrosion Damage of Inner Surface
}

\author{
Natalia Fedorova ${ }^{1, *}$, Yulianna Dimitriadi ${ }^{1}$, and Nikita Ryapolov $^{1}$ \\ ${ }^{1}$ Institute of oil and gas, North-Caucasus federal university, 355035, Kulakova avenue, 16, Stavropol, Russia
}

\begin{abstract}
The detection and the measuring of the developing damages must be accompanied by evaluation of the supporting ability of the design in terms of the increasing size of a defect. If the type of damage is dominant one on the further period of the operation, so the received functional dependences can be used to predict a residual life of the designs. As illustrated by the casing pipes of $168.3 \mathrm{~mm}$ diameter, where the dominant damage is the general corrosion of the inner surface, the algorithm to receive the expression for the calculation of coefficients of the lowering of the supporting ability to the excessive inner pressure in the function of the nominal thickness of pipes wall and corrosion depth is shown. The algorithm of the application of the received expression to calculate the residual life of the damaged casing pipes is given.
\end{abstract}

\section{Introduction}

The monitoring of the technical state is realized not only with the prevention aim of sudden design damage but for the development of the activities to increase their durability.

The prevention of the damages is connected with determination of the actual technical state and parameters values, characterizing the ability of the design to realize the specified functions.

The development of measures to increase durability of the designs is more difficult problem. Its solving consists in analysis, evaluation and information consolidation receiving in the result of instrumental examinations of the diagnostics object. Particularly, it provides for the calculation of the supporting ability of the design in the function of the typical damages size, the detection of mechanism of the developing damage of the design and the change of mechanical metal characteristics and also the establishment of the residual life of the designs.

That was pointed in the 70-s of the last century [1], and it is still a contemporary issue today. This is completely relating to the corrosion damages, particularly, to the pipes of casing strings in the wells (oil, gas and so on).

We have marked that functional dependences of the supporting ability from the typical size of the developing damage can be used to predict a residual life of the design.

2 The algorithm to receive the dependence of the supporting ability of the casing pipes to the excessive inner pressure at the general corrosion of the inner surface
The excessive inner $P^{\prime}{ }_{T}$ pressure at which the maximal stresses in the damaged pipes are equal a yield point of material can be defined according to the formula:

$$
\mathrm{P}_{\mathrm{T}}^{\prime}=\mathrm{P}_{\mathrm{T}} \cdot \mathrm{K}_{2},
$$

where $P_{T}$ - nominal strength characteristics of the pipes; $K_{2}=f(\delta, u)-$ coefficient of the lowering of the supporting ability of the damaged pipes to the inner pressure in the function of the wall thickness $\delta$ and corrosion depth $u$.

Note that the expressions for $K_{2}$ coefficient calculation are individual for every pipes diameter.

To find $K_{2}=f(\delta, \mathrm{u})$ is necessary to develop the calculation scheme of the damaged pipes, to realize the strength calculations, to determine the discrete values of $K_{2}$ at the every thickness of pipes wall and the increasing of size of the damage, also the statistic processing of the obtained data bulks.

\section{Research methods}

The development of the calculation scheme of the damaged pipes was being realized taking into consideration the next factors: corrosion depth, size, geometrical configuration and square of the damage, the change of metal structure and also the presence of regular geometric stress concentrator along the full length of the string (muff threaded connections). Taking into account the static character of the operational loads and the work of the pipes in the casing string in the wells within the limits of elasticity the coefficients $K_{1}$ and $K_{2}$ were taken equal to the reciprocal values of the theoretical coefficients of concentration of the elastic stresses.

The task was analytically accomplished using the software based on the finite elements method [2].

* Corresponding author: fng8@mail.ru 


\section{The calculation scheme for the analytic researches}

Physico-mechanical characteristics (being changed at the corrosion damage) are rigidity of the pipes and also plasticity and strength of metal. Ideology to receive a calculation scheme is the next.

The change of rigidity happening due to the decrease of the wall thickness of the pipe increases the probability of its work with a bend in the places of stresses concentration.

The lowering of plastic metal properties will lead to the loss of its ability to redistribute the local stresses exceeding the yield strength of material due to the inconsiderable plastic deformations. This increases the tendency of metal to the brittle destruction.

In the complex, the decrease of the pipe rigidity and the lowering of the plastic properties of metal mean that its supporting ability will be limited by the stresses condition in the zones of the stress concentration. The geometric stresses concentrator are the zones of muff connections for the pipes (rotational shells) of the casing pipe.

The increased stresses can be analytically calculated using the general theory of shells of rotation [3]. When the work of the casing pipes on the excessive inner pressure happens, the muffs play a role of elements preventing the free pipes enlargement. Herewith the casing pipe can be presented as the design consisting in rotational shells (casing pipes) with the elastic rings on the ends. The task is to calculate the increased stresses happening in the zones of the rings installations. The calculation scheme is illustrated by figure $1, \mathrm{a}, \mathrm{b}, \mathrm{c}, \mathrm{d}$; where 1 - the length of the examining area of the shell, $\delta$ и $\mathrm{R}$ - the wall thickness and the average radius correspondently, $\mathrm{F}$ - the square of cross-section of the ring, $\mathrm{P}_{\text {inner }}$ - the excessive pressure and the inner forces $\mathrm{N}$, arising at this in its cross-sections.

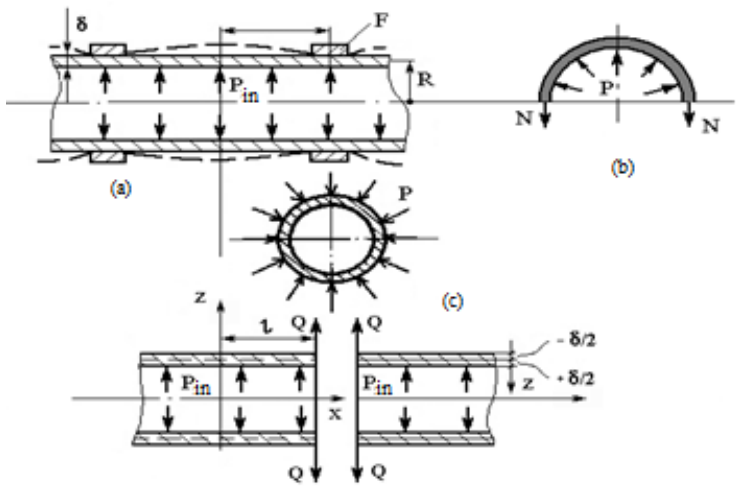

(d)

Fig. 1. The calculation scheme to determine the increased stresses in the zones of muff connections in the casing pipes: aprincipal scheme of the design; $b-a$ half of the ring loading with pressure $P$, acting on it from the side of the shell due to the restraining of its free radial deformation; $\mathrm{c}$ - cross-section of the shell with acting reaction pressure from the side of the ring $\mathrm{P} ; \mathrm{d}$ - equivalent scheme where in the zone of the ring installation, the shell is divided and loaded with edge forces Q.
Maximal stresses are calculated according to the formula:

$$
(\sigma \mathrm{x}) \max =\frac{1,82}{1+\frac{8 \beta^{3} \mathrm{DR}^{2}}{\mathrm{EF}}} \cdot \frac{\mathrm{P}_{\text {inner }} \mathrm{R}}{\delta}
$$

The fraction before the multiplier $\mathrm{P}_{\text {inner }} \mathrm{R} / \delta$ is a coefficient of stresses concentration arising in the shell due to the restraining its free radial deformation by the ring.

\section{The research of the corrosion damage by the finite elements method}

The distribution of the corrosion damage was being modelled on the inner surface and the thickness of pipe wall with diameter $168.3 \mathrm{~mm}, \delta=8 \mathrm{~mm}$. The parameters of model: length - $200 \mathrm{~mm}$, length of the damaged part $100 \mathrm{~mm}$, partitions number on wall thickness -8 , on arcs of the inner and the outer diameter -15 , on pipe length 40. Roughness of the damaged surface was being created by removal of the finite elements in the chessboard manner from the surface layer of metal by thickness in 1 $\mathrm{mm}$. During calculations a depth of the corrosion spot was being changed from 1 to $5 \mathrm{~mm}$, and its square from $30 \%$ to $100 \%$ of the inner pipe surface.

The nominal values of ovality and wall thickness variation of the pipe were not taken into consideration to exclude their influence on calculations results. Besides, the dominant value for the damaged pipe has "wall thickness variation" obtained due to the metal corrosion. The conditions of the attaching of the model have taken into consideration a symmetry of the design and the load.

On figure 2 there is a stress-strain behaviour of the finite element model of the damaged pipe with rough surface of the damage $P_{\text {inner }}=31,6 \mathrm{MPa}$; depth of corrosion $-5 \mathrm{~mm}$. The defect takes $50 \%$ of square of the inner surface.

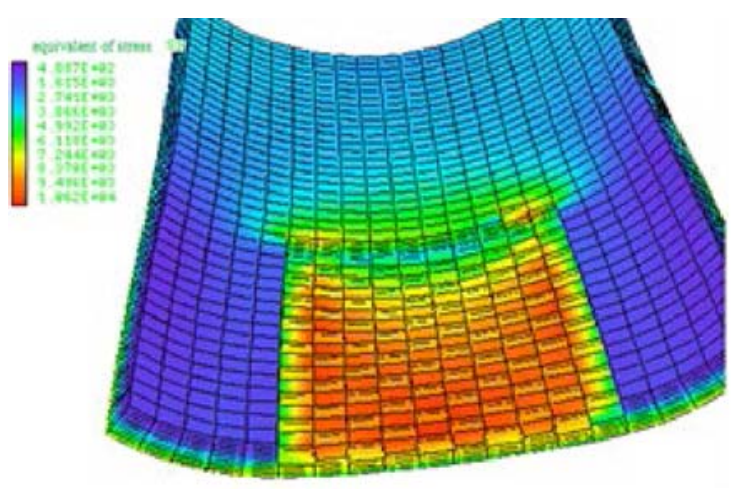

Fig. 2. Stress-strain behaviour of a model of the damaged pipe.

When researches on the finite-elements models were realized, it was received that the less the square of corrosion spot, the higher the stresses in it.

That is, a stress condition promoting its distribution is formed in the produced corrosion spot. With a rise of corrosion spot square, the stresses are declined. When $100 \%$ of the corrosion damage of the inner surface, they 
are minimal. We may consider about the process stabilization.

But if the operating load will exceed the admissible value for the damaged pipe, that will promote a formation of new corrosion spot and damaging of the next metal layer.

Roughness of the surface insignificantly influences on the value of stresses in the zone of the damage.

\section{The researches results of the supporting ability of the damaged pipes to the inner excessive pressure}

On figure 3 you can see the obtained dependences of the coefficients of stresses concentration from the corrosion depth where percentage corresponds to the square of the damaged surface, obtained where modeling of the rough and the smooth surface of the damage; ND - a curve obtained when calculating «coefficients of the stresses concentration» according to the normative document [4] taking into consideration that corrosion as a decrease of thickness of the pipes wall; "loss of rigidity" corresponds to the results of the analytic calculation (calculation scheme on figure 1).

From figure you can see that a graph corresponding to the examination of the corrosion damage as a factor leading to the lowering of physico-mechanic peculiarities of the design creates the upper boundary of the area of values of the stresses concentration coefficient. The lower boundary of the area of its values is a graph obtained taking into consideration that the corrosion damage as an equal decrease of thickness of the pipes wall.

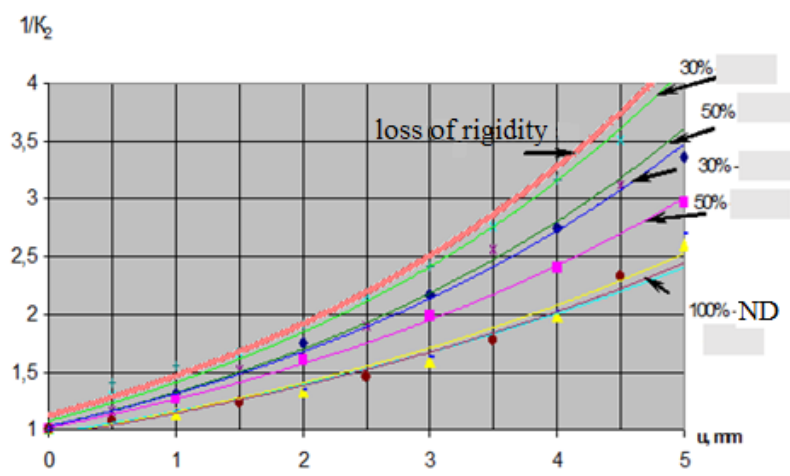

Fig. 3. Dependences of the coefficient of the stresses concentration from the corrosion depth at the different calculation schemes.

Inside the area there are the curves obtained taking into account of the corrosion damage as a geometrical stresses concentrator at the different square of the damage surface. At that a graph that corresponds minimal (from the considered values) square of the damaged surface is getting close to the upper boundary of the values area. The graphs practically coincide with the lower boundary of the area; these graph were obtained at the modeling either of the smooth or the rough surface at $100 \%$ corrosion damage of the inner pipes surface.

\section{Conclusion}

A value model of the corrosion damage as a factor leading to the lowering of design rigidity is a mathematic model of the supporting ability of the damaged casing pipes that work on the excessive inner pressure.

\section{Practical importance}

On the obtained mathematic model for the pipes of diameter series there were received the functional dependences $K_{2}=f(\delta, u)$. For the cemented pipes of the dimeter $168.3 \mathrm{~mm}$ such dependence has the next view:

$$
\mathrm{K} 2=(0,0235 \cdot \delta+0,8038) \cdot \exp (0,0304 \cdot \delta-0,5455) \mathrm{u}
$$

\section{The prediction of the residual life of the damaged pipes}

The expression (3) is a characteristics reflecting a pattern of the lowering of the supporting ability of the $168.3 \mathrm{~mm}$ diameter pipe to the inner pressure the general corrosion damage of the inner surface.

To calculate a residual life $T$ the expression (3) is transformed relatively to $u$ and according to it an maximum permissible corrosion depth for the pipes is calculated:

$$
\begin{gathered}
\mathrm{u}=\frac{\ln \left[\mathrm{K}_{2} /(0,0235 \cdot \delta+0,8038)\right]}{0,0304 \cdot \delta-0,5455}, \\
\mathrm{~K}_{2}=\mathrm{n}_{2} \cdot \mathrm{P}_{\text {excessive.working }} / \mathrm{P}_{\mathrm{T}},
\end{gathered}
$$

where $P_{\text {excessive.working }}$ - operation load (excessive inner pressure);

$n_{2}$ - normative coefficient of margin of safety of pipes to the inner pressure;

$P_{T}-$ normative value of strength characteristics of pipes.

$$
\begin{gathered}
\mathrm{T}=\mathrm{u}^{\prime} / \mathrm{V}, \\
\mathrm{u}^{\prime}=\mathrm{u}-\Delta \delta,
\end{gathered}
$$

where $\Delta \delta-$ a decrease of wall thickness fixed on the moment of calculation realization, $\mathrm{mm}$;

$V$ - corrosion velocity ( $\mathrm{mm} /$ year), corrosion is fixed according to results of diagnostics of the technical state of the casing string and taken linear.

We should mark that an individual residual pipes life is predicted at the same time.

\section{Conclusions}

As may be seen, along with the development of the methods and means of monitoring for the corrosion damages of the designs, it is rational to realize the analytical researches of their supporting ability depending on typical size of the damage.

However, at that it is necessary to decide at what types of the corrosion damages the realization of such kinds of researches makes sense. It seems that it is 
rational to realize if the considering type of corrosion is the dominant mechanism of the damage on predicting period of the designs operation.

\section{References}

1. S.V. Sernsen, V.P. Kogaev, R.M. Shneiderovich, Supporting ability and strength calculations of parts of machines: manual and reference book, 488 (1975)

2. N.G. Fedorova, Supporting ability of casing pipes at general corrosion damage of their inner surface, Petroleum farm, 5, 32-35 (2004)

3. A.M. Kats, Theory of elasticity, 208 (2002)

4. Manual to calculate casing pipes for oil-and-gas wells: instead RD 39-7/1-0001-89 (VNIIGAS, 194, 1997)

5. N.G. Fedorova, Prediction of the service life for casing pipes, Gas industry, 11, 69-72 (2004) 